\title{
A global reaction route mapping-based kinetic Monte Carlo algorithm
}

Izaac Mitchell, Stephan Irle', and Alister J. Page'

Citation: The Journal of Chemical Physics 145, 024105 (2016); doi: 10.1063/1.4954660

View online: http://dx.doi.org/10.1063/1.4954660

View Table of Contents: http://aip.scitation.org/toc/jcp/145/2

Published by the American Institute of Physics

\section{Articles you may be interested in}

Carbon membranes for efficient water-ethanol separation

The Journal of Chemical Physics 145, 124708124708 (2016); 10.1063/1.4963098

Effective scheme for partitioning covalent bonds in density-functional embedding theory: From molecules to extended covalent systems

The Journal of Chemical Physics 145, 244103244103 (2016); 10.1063/1.4972012

Overview: Experimental studies of crystal nucleation: Metals and colloids

The Journal of Chemical Physics 145, 211703211703 (2016); 10.1063/1.4963684

Relaxation times in deformed polymer glasses: A comparison between molecular simulations and two theories

The Journal of Chemical Physics 145, 064505064505 (2016); 10.1063/1.4960208

Nonequilibrium diagrammatic technique for Hubbard Green functions

The Journal of Chemical Physics 146, 092301092301 (2016); 10.1063/1.4965825

The melting of stable glasses is governed by nucleation-and-growth dynamics

The Journal of Chemical Physics 144, 244506244506 (2016); 10.1063/1.4954327

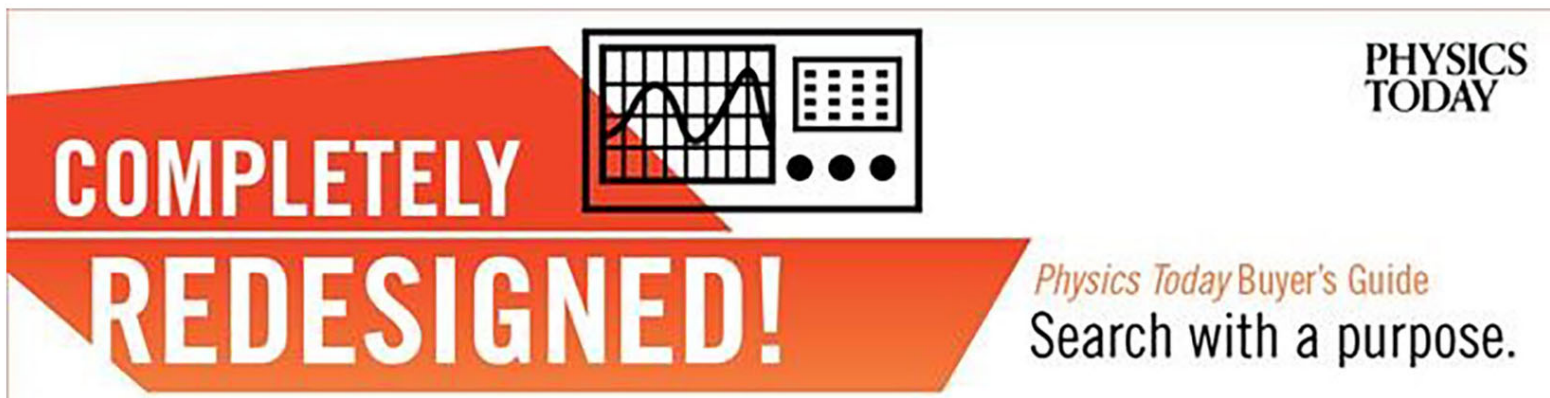




\title{
A global reaction route mapping-based kinetic Monte Carlo algorithm
}

\author{
Izaac Mitchell, ${ }^{1}$ Stephan Irle, ${ }^{2, a)}$ and Alister J. Page ${ }^{1, a)}$ \\ ${ }^{1}$ Newcastle Institute for Energy and Resources, The University of Newcastle, Callaghan 2308, Australia \\ ${ }^{2}$ Institute of Transformative Bio-Molecules (WPI-ITbM) and Department of Chemistry, \\ Graduate School of Science, Nagoya University, Nagoya 464-8602, Japan
}

(Received 25 April 2016; accepted 11 June 2016; published online 12 July 2016)

\begin{abstract}
We propose a new on-the-fly kinetic Monte Carlo (KMC) method that is based on exhaustive potential energy surface searching carried out with the global reaction route mapping (GRRM) algorithm. Starting from any given equilibrium state, this GRRM-KMC algorithm performs a one-step GRRM search to identify all surrounding transition states. Intrinsic reaction coordinate pathways are then calculated to identify potential subsequent equilibrium states. Harmonic transition state theory is used to calculate rate constants for all potential pathways, before a standard KMC accept/reject selection is performed. The selected pathway is then used to propagate the system forward in time, which is calculated on the basis of 1st order kinetics. The GRRM-KMC algorithm is validated here in two challenging contexts: intramolecular proton transfer in malonaldehyde and surface carbon diffusion on an iron nanoparticle. We demonstrate that in both cases the GRRM-KMC method is capable of reproducing the 1 st order kinetics observed during independent quantum chemical molecular dynamics simulations using the density-functional tight-binding potential. Published by AIP Publishing. [http://dx.doi.org/10.1063/1.4954660]
\end{abstract}

\section{INTRODUCTION}

Two of the most common methods for simulating chemical equilibria and non-equilibrium processes are molecular dynamics (MD), ${ }^{1,2}$ a purely deterministic method based on the classical laws of motion, and the Monte Carlo (MC) method, ${ }^{3,4}$ a method which samples the local potential energy surface (PES) on a stochastic basis. Both $\mathrm{MC}$ and $\mathrm{MD}$ can predict the time-averaged properties of atomic and molecular ensembles. In both cases the accuracy with which these properties can be determined is limited by how efficiently the electronic energy of the system can be calculated. MD can also predict the time evolution of atomic ensembles, but is limited by how efficiently the interatomic forces can be calculated at each simulation step. This limits the time scale of these methods, and hence, what chemical processes they can meaningfully simulate. ${ }^{5}$ Hybrid MD/MC methods and force bias MC methods also have this limitation. Molecular dynamics is restricted further by the requirement that the time integration step must be sufficiently small so that nuclear motion over the PES is integrated smoothly, thus avoiding unrealistic dynamics. This is particularly the case for chemically reactive systems. A commonly quoted heuristic is that the time step must be (at most) $50 \%$ of the highest frequency vibrational mode present in the system. There have been several attempts aimed at overcoming this "time scale problem." Notable advances include force biased $^{5,6}$ and time-stamped force biased ${ }^{7} \mathrm{MC}$ methods, parallel replica dynamics, ${ }^{8}$ umbrella sampling, ${ }^{9-12}$ hyperdynamics, ${ }^{13}$ temperature-accelerated dynamics and bond-boosting

\footnotetext{
a) Authors to whom correspondence should be addressed. Electronic addresses: sirle@chem.nagoya-u.ac.jp and alister.page@newcastle.edu.au
}

methods, ${ }^{14}$ and collective variable-driven hyperdynamics (CVHD). ${ }^{15}$

Amongst the most versatile solutions to the time scale problem, and in particular the problem of rare events, are on-the-fly kinetic MC (KMC) methods. ${ }^{16}$ Conventional MC methods simulate state-to-state dynamics by randomly moving atomic nuclei on the local PES, and either accepting or rejecting the new nuclear configuration according to some predetermined criteria, usually energy. On the other hand, kinetic MC (KMC) achieves time evolution by choosing one among a set of possible "moves," i.e., state-to-state transitions, at random with a probability that is proportional to the associated rate constant. ${ }^{16}$ Provided that this "movetable" exhaustively spans the complexity of the system, $\mathrm{KMC}$ is capable of efficiently propagating so-called rare-event dynamics well beyond the capabilities of traditional MD or MC. KMC was initially developed in the 1960 s for modelling vacancy migration simulations in alloys. ${ }^{17}$

The key limitation of KMC in this respect is that a complete set of state-to-state transitions and their associated rate constants must be known in advance. This means that traditional KMC is incapable of simulating emergent phenomena, such as self-assembly. On-the-fly $\mathrm{KMC}$, on the other hand, determines state-to-state transitions based on the nature of the local PES around a particular local minimum, at each state of the system. ${ }^{18}$ While on-the-fly KMC removes the need for an a priori move-table, it requires a method of finding all local transition states (TSs). This in itself is a tremendous task.

A number of TS search algorithms have been previously reported, and such methods are now routine in popular quantum chemistry programs. These algorithms typically fall into two categories: (1) double-ended searches, for which a TS connecting two local minima on the PES is the target (e.g., the nudged-elastic band ${ }^{19}$ and quasi-synchronous transit ${ }^{20}$ 
methods) and (2) single-ended searches, which search for TSs from a single local minimum on the PES (e.g., eigenvector following methods ${ }^{21,22}$ ). In the context of on-the-fly $\mathrm{KMC}$, both approaches have shortcomings: double-ended searches, by construction, require knowledge of both equilibrium states in order to identify the TS between them (and hence the kinetic parameters of the transition); and single-ended searches find only a single TS, and so are not guaranteed to accurately describe the evolution of the system.

Instead, on-the-fly KMC requires a PES search algorithm that can exhaustively search the region surrounding a local minimum. Global reaction route mapping (GRRM), ${ }^{23-26}$ which is based on the anharmonic downward distortion following (ADDF) and intrinsic reaction coordinate (IRC) search algorithms, ${ }^{23,25,27,28}$ is one such method. Given a local minimum on the PES, GRRM is capable of locating all TS structures connected to it by searching for minima on a scaled energy hypersphere, which correspond to ADDs on the true molecular PES that signify a TS. An IRC pathway is then calculated at each TS to determine all connected local minima. By repeating this process enough times, GRRM is capable of exhaustively searching the entire molecular PES (typically within a prescribed energy threshold).

In this work, we present a new on-the-fly KMC algorithm that utilises GRRM ${ }^{24,26-28}$ to exhaustively search for local TSs on the PES around an arbitrary local minimum. Although GRRM can be used as a basis for exhaustively searching for all stationary points on a molecular PES, our approach truncates the GRRM search to a single local minimum. The TSs identified by the truncated GRRM search are then used to construct an on-the-fly move table, which is applied during a subsequent KMC time propagation step. We demonstrate the efficacy of this algorithm on model systems pertinent to two archetypal chemical reactions-intramolecular proton transfer and nanoscale surface diffusion.

\section{COMPUTATIONAL METHODS}

\section{A. GRRM-based on-the-fly kinetic Monte Carlo algorithm}

Our on-the-fly KMC algorithm is depicted schematically in Figure 1. We refer to this method as "GRRM-KMC" from this point onwards. Starting from the $i$ th equilibrium state, $\mathrm{EQ}_{i}$, the algorithm consists of the following steps:

1. A one-step GRRM search is performed to identify all $n$ TS structures $\left(\mathrm{TS}_{i}^{j}, 1 \leq j \leq n\right)$ connected to $\mathrm{EQ}_{i}$.

2. IRC pathways are calculated for all $\mathrm{TS}_{i}^{j}$ to determine potential new equilibrium states $\mathrm{EQ}_{i+1}^{j}$. $\mathrm{EQ}_{i+1}^{j}$ structures are obtained by geometry optimisation of the final IRC point.

3. For all reactive pathways, rate constants $k_{i}^{j}$ are calculated assuming 1st-order kinetics using harmonic transition state theory (HTST) via Vineyard theory, ${ }^{29}$

$$
k_{i}^{j}=A_{i}^{j} \exp \left(-\frac{\Delta E_{i}^{j}}{k_{b} T}\right) .
$$

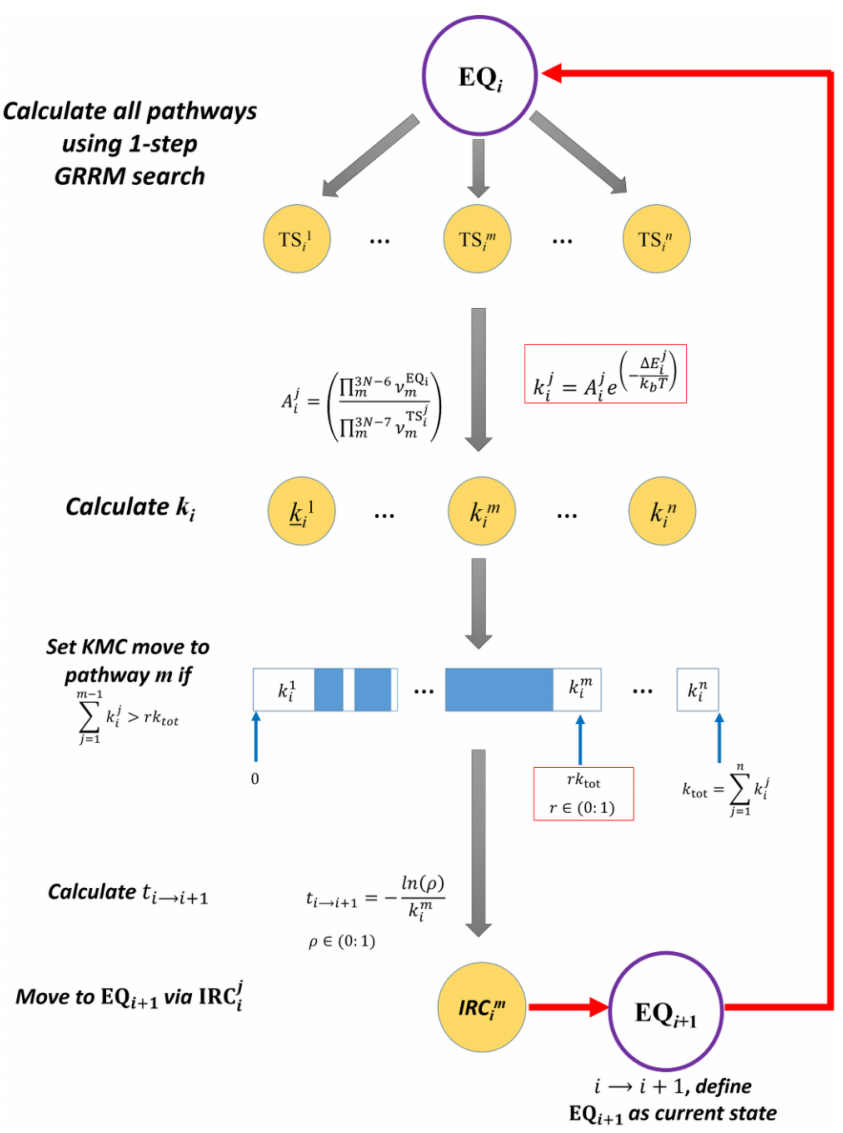

FIG. 1. Schematic representation of the GRRM-based on-the-fly KMC algorithm. Initially GRRM is used to find all transitions states $\mathrm{TS}_{i}^{j}$ around an equilibrium state $\mathrm{EQ}_{i}$. Activation barriers $\Delta E_{i}^{j}$ are used to calculate rate constants $k_{i}^{j}$ on the basis of 1 st order kinetics. $\mathrm{TS}_{i}^{m}$ is selected with a probability proportional to the rate constant $k_{i}^{m}$ and the total sum of the rate constants $k_{\text {tot }}$. A time step is calculated using Equation (5). $\mathrm{TS}_{i}^{m}$ is used to find the next $\mathrm{EQ}, \mathrm{EQ}_{i+1}^{m}$ via the IRC between the two equilibrium states.

Here $k_{B}$ is Boltzmann's constant, $T$ is the temperature, $\Delta E_{i}^{j}$ is the activation energy for $\mathrm{TS}_{i}^{j}$ relative to $\mathrm{EQ}_{i}$. We note here that $\Delta H_{i}^{j}$, i.e., the activation enthalpy for $\mathrm{TS}_{i}^{j}$ relative to $\mathrm{EQ}_{i}$, can also be used to calculate $k_{i}^{j}$ in cases where thermal contributions (i.e., $k_{b} T$ ) to the total internal energy become significant. The exponential prefactors $A_{i}^{j}$ for $\mathrm{EQ}_{i}$ and $\mathrm{TS}_{i}^{j}$ are calculated using

$$
A_{i}^{j}=\left(\frac{\prod_{m}^{3 N-6} v_{m}^{E Q_{i}}}{\prod_{m}^{3 N-7} v_{m}^{T S_{i}^{j}}}\right),
$$

where $v_{m}^{E Q_{i}}$ is the $m$ th vibrational mode of $\mathrm{EQ}_{i}$ and $v_{m}^{T S_{i}^{j}}$ is the $m$ th (real) vibrational mode of $\mathrm{TS}_{i}^{j}$.

4. Rate constants $k_{i}^{j}(1 \leq j \leq n)$, are summated to yield $k_{\text {tot }}$,

$$
k_{\text {tot }}=\sum_{j=1}^{n} k_{i}^{j}
$$

5. $k_{\text {tot }}$ is multiplied by a random number $r \in[0,1]$. The $m$ th pathway is chosen for the KMC step between states $\mathrm{EQ}_{i}$ and $\mathrm{EQ}_{i+1}$ for the single value of $m$ such that

$$
\sum_{j=1}^{m-1} k_{i}^{j}>r k_{\mathrm{tot}}
$$


6. The time associated with the $\mathrm{EQ}_{i} \rightarrow \mathrm{EQ}_{i+1}^{m}$ transition via $\mathrm{TS}_{i}^{m}$ is defined as

$$
t_{i \rightarrow i+1}=-\frac{\ln (\rho)}{k_{i}^{m}}
$$

for a second independent random number $\rho \in[0,1]$.

7. $\mathrm{EQ}_{i+1}^{m}$ is defined as the current state of the system, i.e., $\mathrm{EQ}_{i+1}$, and the algorithm returns to step (1).

\section{B. Quantum chemical methods}

In Sec. III, we demonstrate the utility of the GRRMKMC algorithm in two contrasting contexts. The first is a fast intramolecular process (proton transfer in malonaldehyde, Section III A), whereas the second is a far slower process (surface diffusion on iron nanoparticles, Section III B). These comparisons are based on a variety of different electronic structure methods.

Density functional tight binding (DFTB) is used for all quantum chemical MD simulations reported in this work. The DFTB method ${ }^{30-38}$ is an extended Hückel method parameterised using generalised-gradient approximation density functional theory (GGA-DFT), ${ }^{31}$ which is based on the Foulkes-Haydock ansatz. This ansatz assumes that the electronic density $\rho$ can be expressed as a reference density $\rho_{0}$ plus a small density perturbation (i.e., $\rho=\rho_{0}+\Delta \rho$ ). Under this assumption, it is possible to expand the GGA-DFT exchangecorrelation potential as a Taylor series,

$$
\begin{aligned}
E= & \sum_{i}^{\text {valence }} n_{i} \varepsilon_{i}+\frac{1}{2} \sum_{A, B}^{\text {atoms }} E_{A B}^{\text {rep }}+\frac{1}{2} \sum_{A, B}^{\text {atoms }} \gamma_{A B} \Delta q_{A} \Delta q_{B} \\
& +\frac{1}{3} \sum_{A, B}^{\text {atoms }} \Gamma_{A B} \Delta q_{A}^{2} \Delta q_{B}+\cdots
\end{aligned}
$$

in which the 1st and 2nd terms are the electronic and repulsive terms, respectively. The electronic term is expressed in terms of orbital occupations and energies $n_{i}$ and $\varepsilon_{i}$, respectively. The 3rd term is the 2 nd order correction to the DFTB energy, ${ }^{36}$ which models charge transfer between atoms $A$ and $B$ in terms of the Hubbard parameter $\gamma_{A B}$. This correction is necessary for systems which exhibit heterogeneous bonding (so-called self-consistent-charge DFTB, or SCC-DFTB). The 4th term is the 3rd order correction to DFTB, ${ }^{35}$ which describes how the reference density $\rho_{0}$ for each atom responds to the local chemical environment, in terms of the Hubbard parameter and its derivative, $\Gamma_{A B}$.

All DFTB calculations reported here were made using the DFTB + program, ${ }^{39}$ and utilised an electronic temperature of $10000 \mathrm{~K}$. While this is higher than necessary for small molecules such as malonaldehyde, it is employed here in order to guarantee SCC-DFTB convergence during QM/MD and GRRM-KMC simulations of surface diffusion on $\mathrm{Fe}$ nanoparticles (Section III B). For the sake of consistency, the same electronic temperature is employed in both contexts.

DFT and 2nd order Møller-Plesset perturbation theory (MP2) were also employed to study intramolecular proton transfer in malonaldehyde using GRRM-KMC (Section III A). The M06-2X Minnesota exchange-correlation functional was employed, as it has been previously shown to accurately describe proton affinities. ${ }^{40,41}$ MP2, which explicitly accounts for dynamic electron correlation, was employed due to its greater accuracy in the context of proton transfer. ${ }^{4-44}$ Both M06-2X and MP2 methods were used in conjunction with an aug-cc-PVTZ basis set. ${ }^{45,46}$ All DFT and MP2 calculations reported here were calculated using Gaussian $09 .{ }^{47}$

\section{RESULTS AND DISCUSSION}

\section{A. Intramolecular proton transfer in malonaldehyde}

Proton transfer in malonaldehyde is an archetypal intramolecular process, and the reactive PES for this process has been studied extensively (see Refs. 48-50 and references therein). Intramolecular proton transfer in malonaldehyde is an ideal benchmark for the GRRM-KMC algorithm. It is simple in that the transfer kinetics are determined by only a single reactive barrier (Figure 2). This simplifies the GRRM search in step (1) of the GRRM-KMC algorithm. However, the mechanism of proton transfer is complex and includes quantum tunneling effects, thermally activated reactions, and barrier vanishing reactions. ${ }^{48}$ The nature of the reaction PES is such that frequent re-crossing events (processes in which an initial proton transfer event triggers a subsequent transition immediately afterwards) are observed. ${ }^{48,49}$ These recrossing events pose a particular challenge to ergodic KMC methods (discussed below). Previous studies have predicted proton transfer rates of $0.27 \mathrm{ps}^{-1}$ in vacuum, ${ }^{49}$ while neon and water solvents lead to rates of 0.89 and $0.70 \mathrm{ps}^{-1}$, respectively. ${ }^{48}$

We first comment on the efficacy of DFTB3 in describing the intramolecular proton transfer reaction. GRRM correctly predicts the intramolecular proton transfer pathway in malonaldehyde, using DFTB3, M06-2X, and MP2 levels of theory. Figure 2 compares these DFTB3/3ob-3-1 results with M06-2X/aug-cc-pVTZ and MP2/aug-cc-pVTZ results. The $\mathrm{O}-\mathrm{H}$ bond in the local minimum (Figure 2, left and right) is 1.004, 0.992, and $1.002 \AA$, respectively, with these methods. In the TS structure, DFTB3 predicts an $\mathrm{O}-\mathrm{H}$ bond length of $1.196 \AA$, while M06-2X and MP2 predict 1.203 and $1.205 \AA$. The activation energies $\left(\Delta E^{\ddagger}\right)$ and free energies $\left(\Delta G^{\ddagger}\right)$ using DFTB3 are 8.9 and $-0.4 \mathrm{~kJ} \mathrm{~mol}^{-1}$, slightly underestimating the M06-2X/aug-cc-pVTZ values $\left(12.5,4.0 \mathrm{~kJ} \mathrm{~mol}^{-1}\right)$ and MP2/aug-cc-pVTZ values $\left(11.5,2.7 \mathrm{~kJ} \mathrm{~mol}^{-1}\right)$. Thus, in

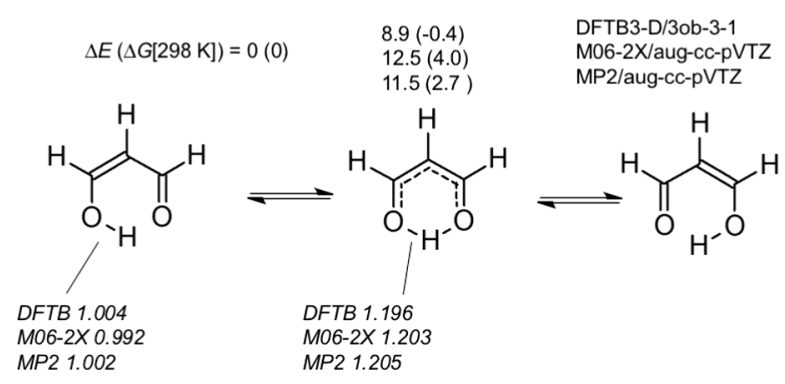

FIG. 2. Intramolecular proton transfer in malonaldehyde, calculated using DFTB3/3ob-3-1, M06-2X/aug-cc-pVTZ, and MP2/aug-cc-pVTZ. Local minima are left and right, and the proton transfer TS is centre. Bond lengths (italics) given in Angstrom, activation energies given in $\mathrm{kJ} / \mathrm{mol}$. 
general, the structural and energetic agreement between DFTB3 and these more reliable methods is acceptable. While the DFTB3/3ob-3-1 $\Delta E^{\ddagger}$ value is positive, the $\Delta H^{\ddagger}$ value (not shown in Figure 2) is $-2.8 \mathrm{~kJ} \mathrm{~mol}^{-1}$, due to the $\Delta H$ correction terms within the harmonic approximation. For this reason, all subsequent GRRM-KMC reported here are based on $\Delta E^{\ddagger}$, not $\Delta H^{\ddagger}$.

Constant-temperature (NVT) DFTB3/MD simulations were used to benchmark GRRM-KMC results for intramolecular proton transfer in malonaldehyde. The 3rd-order correction in DFTB3 (term 4, Equation (6)), in conjunction with the 3ob parameter sets, ${ }^{51-54}$ enables an accurate treatment of hydrogen bonding, ${ }^{53}$ which is crucial in the context of intramolecular proton transfer. These DFTB3/MD simulations employed an initial temperature of $298 \mathrm{~K}$. The velocity Verlet algorithm ${ }^{55}$ was used to propagate the classical equations of motion, using a time step of $0.1 \mathrm{fs}$. This timestep is sufficiently small to ensure an accurate description of proton transfer. Nuclear temperature was maintained at $298 \mathrm{~K}$ using a Nosé-Hoover chain thermostat (chain length 3$)^{56,57}$ coupled to the degrees of freedom of the system. To ensure reliable statistical sampling, 50 independent trajectories were calculated. Each trajectory began with a 10 ps period of equilibration, before proton transfer was analysed over a subsequent period of 290 ps. This resulted in a total of 1653 proton transfer events being observed.

A comparison between proton transfer frequencies as a function of time, calculated using NVT MD and GRRM$\mathrm{KMC}$, is made in Figure 3. Below $1 \mathrm{ps}$, an anomalously high transition frequency is observed in Figure 3(a), consistent with previous reports. ${ }^{48,49}$ This is due to the presence of re-crossing events in the DFTB3/MD simulation and results in a non-exponential decay in the transition frequency at short time scales. ${ }^{48,49}$ These results demonstrate that re-crossing events can be predicted in malonaldehyde using MD in the absence of quantum effects (viz., barrier vanishing and proton tunnelling). By contrast, the decay in transition frequency shown in Figure 3(a) for the GRRM-KMC simulations lacks this non-exponential character at short time scales. These results reflect the fact that deterministic MD can describe re-crossing in malonaldehyde proton transfer (albeit here without describing quantum effects such as proton tunnelling), since the dynamics are propagated directly on the molecular PES. However, GRRM-KMC (and KMC in general) assumes ergodicity; that is, the time interval separating each state-tostate transition is sufficiently large to enable the molecule to equilibrate. Some discrepancy between KMC and MD descriptions of proton transfer kinetics should naturally be anticipated for very fast transitions.

Figure 3(b) corroborates this conclusion; by removing the fast re-crossing events (all transitions faster than 1 ps), a much closer alignment between NVT MD and GRRM-KMC transition distributions is observed. For longer time scale transitions (i.e., $>1$ ps), both Figures 3(a) and 3(b) show much closer agreement between NVT MD and GRRM-KMC transition frequency distributions. The decay in transition frequency is in near perfect agreement here for the two methods. Moreover, the absolute transition frequencies themselves for the GRRM-KMC method are
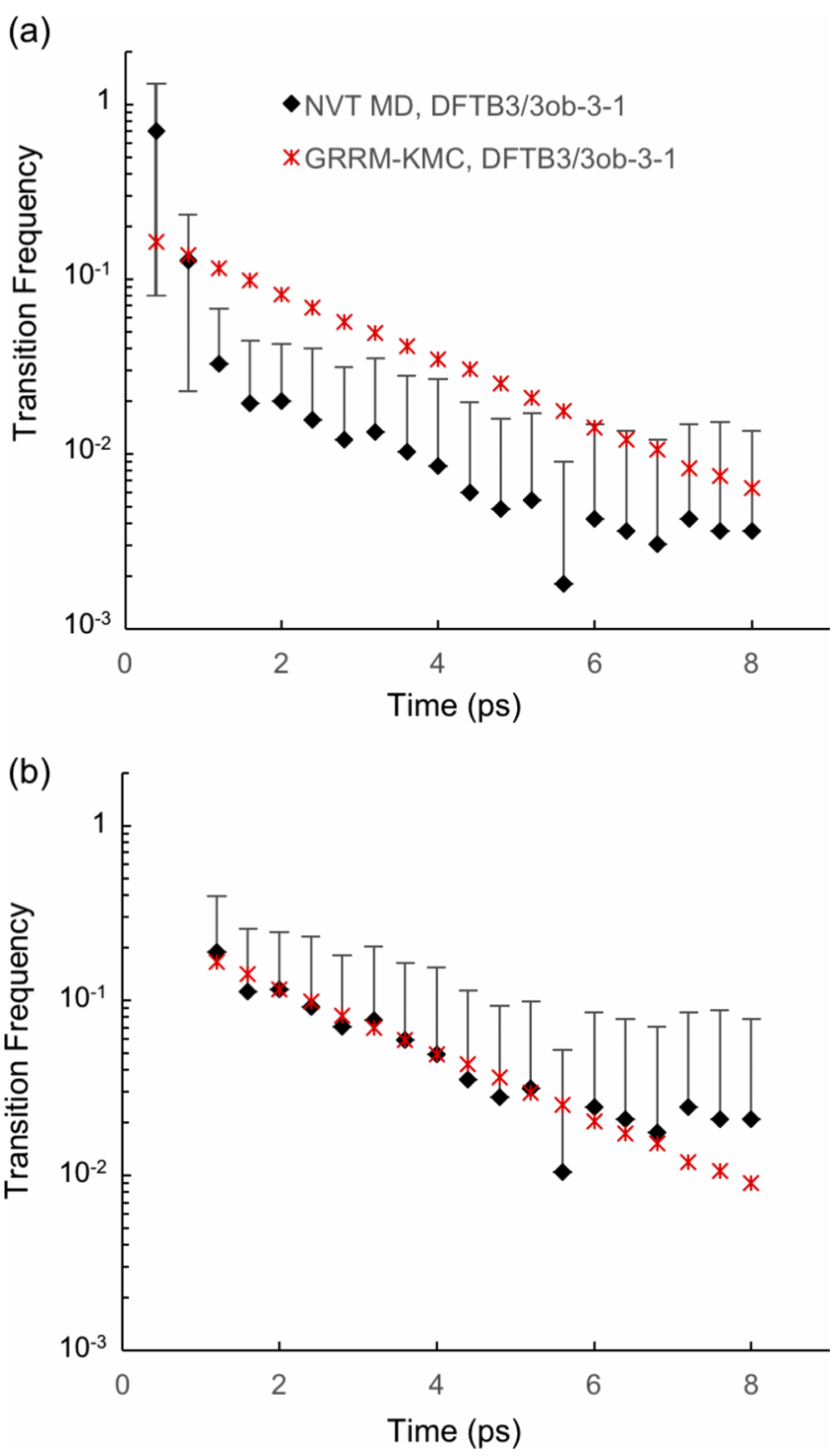

FIG. 3. Normalised frequencies of intramolecular proton transfer in malonaldehyde at $298 \mathrm{~K}$, predicted using NVT MD ( $)$ and GRRM-KMC $(\times)$ : (a) includes all transfer events, including re-crossings faster than $1 \mathrm{ps}$; (b) excludes transitions faster than 1 ps. Both MD and KMC simulations are performed using the DFTB3/3ob-3-1 method. MD simulations predict a non-exponential decay in transition frequency for transitions $<2$ ps. Error bars on MD data points show 1 standard deviation (negative error bars omitted due to logarithmic scale).

in close agreement with those obtained using NVT MD. The average transition rates calculated using NVT MD and GRRM-KMC methods are, respectively, 0.25 and $0.26 \mathrm{ps}^{-1}$. This is consistent with previously reported values in vacuum (discussed above), which is expected due to the accuracy of the DFTB3/3ob-3-1 description of the proton transfer reactive PES, shown in Figure 2.

\section{B. Surface carbon diffusion on an iron nanoparticle}

Our principle motivation for the development of the GRRM-KMC algorithm is the ability to simulate emergent phenomena, such as self-assembly, as accurately and efficiently as possible. Such phenomena can, in principle, be simulated and described using MD. Most often, however, 
the time scale problem limits the utility of MD in this context. Conversely, MC and KMC, by construction, are incapable of describing emergent phenomena all together. Surface diffusion is a phenomenon that fundamentally underpins myriad processes, notably chemical vapour deposition, catalysis, and nanoscale self-assembly. Hence, in the present work, we focus on validating the GRRM-KMC algorithm for a simple surface diffusion model consisting of a carbon atom on an icosahedral $\mathrm{Fe}_{13}$ nanoparticle.

An exhaustive GRRM search of surface carbon atom diffusion on $\mathrm{Fe}_{13}$ (in conjunction with SCC-DFTB/trans3d, see below) yielded the two local minima and one TS shown in Figure 4(a). While this is a remarkably simple PES for a diffusion process, it is unsurprising due to the icosahedral symmetry of the $\mathrm{Fe}_{13}$ nanoparticle. The most stable of these two minima (EQ1) consists of the carbon atom adsorbed symmetrically over the trigonal face of the $\mathrm{Fe}_{13}$ icosahedron, with $\mathrm{Fe}-\mathrm{C}$ bond distances of $2.13 \AA$. The three $\mathrm{Fe}-\mathrm{Fe}$ bonds forming the trigonal face remain intact, with distances of $2.60 \AA$. The other minimum structure (EQ2) consists of the carbon atom intercalating between two iron atoms that form a vertex of the $\mathrm{Fe}_{13}$ icosahedron. Here the $\mathrm{Fe}-\mathrm{Fe}$ bond is cleaved (with a distance of $3.10 \AA$ ), and the $\mathrm{C}-\mathrm{Fe}$ bond lengths are $2.00 \AA$. Consequently EQ2 is $\sim 36 \mathrm{~kJ} \mathrm{~mol}^{-1}$ higher in energy, compared to EQ1. The TS structure linking EQ1 and EQ2 is a late TS, which is structurally and energetically very similar to EQ2. The Fe-Fe vertex bond in TS is elongated (3.03 $\AA$ ) and the $\mathrm{C}-\mathrm{Fe}$ bond lengths are $2.01 \AA$. The activation energy for $\mathrm{EQ} 1 \rightarrow \mathrm{EQ} 2$ carbon diffusion is $36.7 \mathrm{~kJ} \mathrm{~mol}^{-1}$ (i.e., only $0.7 \mathrm{~kJ} \mathrm{~mol}^{-1}$ higher in energy than EQ2).

NVT MD was employed to study surface diffusion of atomic carbon on an $\mathrm{Fe}_{13}$ nanoparticle. The SCC-DFTB method, ${ }^{36}$ in conjunction with the trans $3 \mathrm{~d}$ parameter set, ${ }^{58}$ was used to calculate energies and gradients on-the-fly at each MD step. A Nosé-Hoover chain thermostat ${ }^{56,57}$ (chain length 3) was coupled to the degrees of freedom of the system at a temperature of $1500 \mathrm{~K}$, and the classical equations of motion were propagated using the velocity Verlet algorithm $(\Delta t=1 \mathrm{fs}) .{ }^{55}$ An electronic temperature of $10000 \mathrm{~K}$ was used throughout these simulations. High electronic temperature is often used for Fe-rich systems, ${ }^{59,60}$ since the high density of $d$-orbital states near the Fermi level leads to problems with the convergence of Equation (6) with respect to the nuclear charges $\Delta q .30$ independent trajectories were computed, each consisting of $100 \mathrm{ps}$, which produced 11668 surface diffusion events.

The normalised transition frequencies (for a face-to-face, i.e., EQ1 $\rightarrow$ TS $\rightarrow$ EQ1, diffusion event) computed using NVT MD and GRRM-KMC are compared in Figure 5. From Figure 5(a), it is immediate that the NVT MD transition distribution is non-exponential for transition times $<2$ ps and thus resembles NVT MD data in Figure 3(a) for intramolecular proton transfer in malonaldehyde. Observations from NVT MD trajectories revealed that this non-exponential behaviour resulted from one or more EQ1 $\rightarrow$ TS $\rightarrow$ EQ1 diffusion events following an initial EQ1 $\rightarrow$ TS $\rightarrow$ EQ1 transition. It is likely that this is made possible by the increased kinetic energy of the carbon atom in the vicinity of the TS structure, which increases the likelihood of repeated EQ1 $\rightarrow$ TS $\rightarrow$ EQ1 diffusion events. We also note that EQ2 (Figure 4(a)) was never observed in NVT MD simulations, presumably due to its structural and energetic similarity to TS. Consequently, EQ2 $\rightarrow$ TS $\rightarrow$ EQ1 transitions were disregarded in our GRRM-KMC simulations.

As noted in our discussion of intramolecular proton transfer in malonaldehyde, the GRRM-KMC algorithm assumes ergodicity and so is not expected to accurately describe the frequency of fast EQ1 $\rightarrow$ TS $\rightarrow$ EQ1 diffusion events. Moreover, due to high frequency of these events observed in NVT MD simulations, the latter distribution is biased, which limits agreement between transition frequencies observed with NVT MD and GRRM-KMC methods for longer-scale transitions (i.e., $>2$ ps). Excluding diffusion events faster than 2 ps (Figure 5(b)) leads to near-perfect agreement between the two methods and validates the GRRM-KMC method for simulating long-time scale surface diffusion phenomena. Comparison of transitions occurring on time scales longer than 10 ps was not possible, since no transitions were observed on these time scales in the NVT MD simulations. This illustrates the time scale problem precisely, as recently reported CVHD simulations of carbon diffusion on copper substrates do. ${ }^{15}$ However, 100000 iterations of the GRRM-KMC algorithm (albeit with only a single initial PES (a)

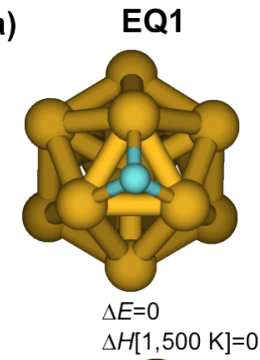

(b)

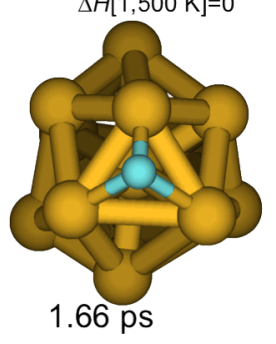

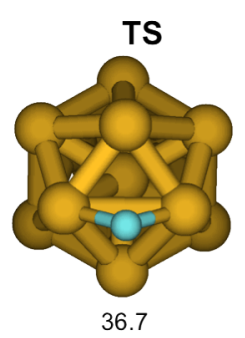

24.2

(c)

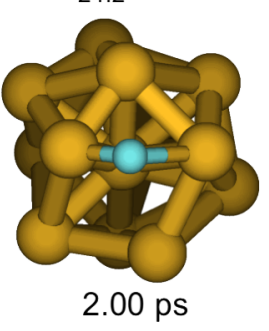

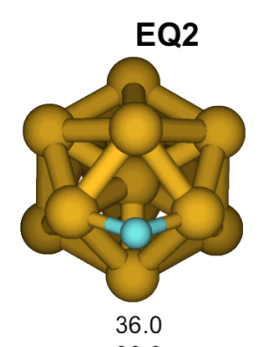

36.0

(d)

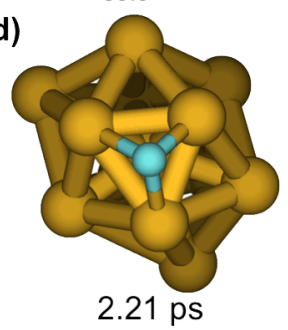

FIG. 4. (a) GRRM-derived local minima and TS structures for carbon diffusion on $\mathrm{Fe}_{13}$ nanoparticle. $\Delta E / \Delta H$ values given in $\mathrm{kJ} \mathrm{mol}^{-1}$. (b)-(d) show a carbon diffusion event observed during NVT MD simulation, corresponding to a EQ1 $\rightarrow$ TS $\rightarrow$ EQ1 transition. Structure EQ2 was not observed during NVT $\mathrm{MD}$, as it is metastable and readily converts back to $\mathrm{EQ1}$ via $\mathrm{TS}$. 
(a)
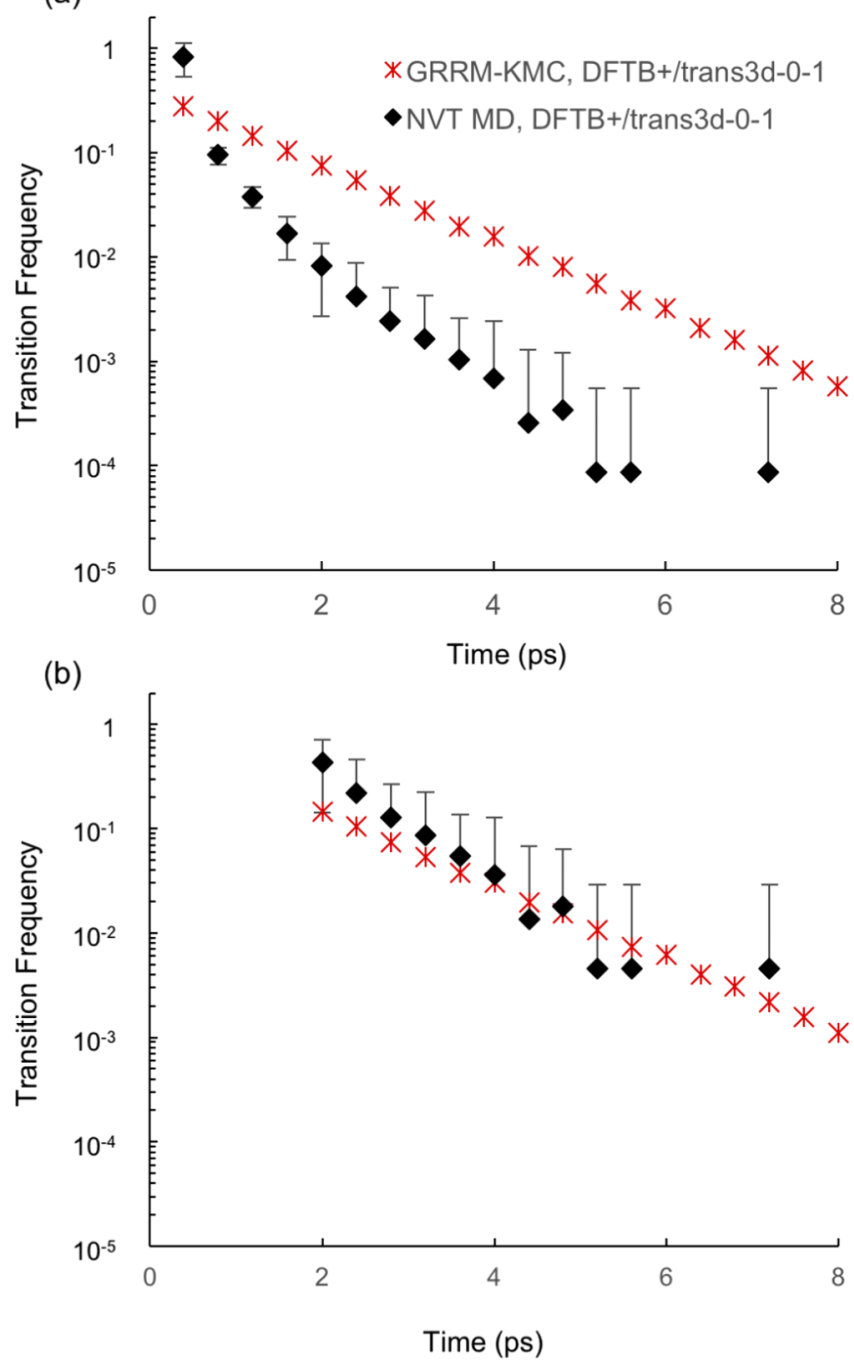

FIG. 5. Normalised frequencies of EQ1 $\rightarrow \mathrm{TS} \rightarrow \mathrm{EQ} 1$ diffusion events for a carbon atom on $\mathrm{Fe}_{13}$ nanoparticle at $1500 \mathrm{~K}$, predicted using NVT MD ( ) and GRRM-KMC ( $\times$ ): (a) includes all transfer events, including transitions faster than $2 \mathrm{ps}$; (b) excludes transitions faster than $2 \mathrm{ps}$. Both MD and KMC simulations are performed using the DFTB3/trans3d-0-1 method. GRRM-KMC transition frequencies are calculated on an enthalpic basis to account for thermal contributions at $1500 \mathrm{~K}$. Error bars on MD data points show 1 standard deviation (negative error bars omitted due to logarithmic scale).

search made possible by the simple nature of the diffusion PES in this case) yielded transitions taking place over time scales of up to $12 \mathrm{ps}$, far beyond what can be reasonably achieved with current $\mathrm{QM} / \mathrm{MD}$ simulations.

\section{CONCLUSION}

We have presented a new on-the-fly KMC algorithm, based on the GRRM PES searching method, and verified its efficacy in two challenging and contrasting applications. For the first of these (intramolecular proton transfer in malonaldehyde), our GRRM-KMC algorithm successfully reproduced the 1st order kinetics observed during independent QM/MD simulations, based on the quantum chemical DFTB3 method. As anticipated, deviations from 1st order kinetics could not be accounted for within the KMC formalism. For intramolecular malonaldehyde proton transfer, this deviation is observed for very fast proton transfer events (i.e., those on time scales $<1 \mathrm{ps}$ ), which arise due to effects such as barrier vanishing reactions and quantum tunnelling. In the absence of these phenomena, GRRM-KMC is capable of reproducing the proton transfer dynamics in this molecule, generally to within the statistical error observed during our QM/MD simulations. The GRRM-KMC method also reproduced the kinetics observed during QM/MD simulations of high-temperature surface diffusion of carbon on an iron nanoparticle. As for the malonaldehyde system, surface carbon diffusion also displays non-1st order kinetic behaviour for very fast (i.e., $<2$ ps) diffusion events. In this case, however, this deviation occurs due to the presence of a late TS associated with the diffusion pathway, which enables successive diffusion events over the nanoparticle surface. Despite this, the GRRM$\mathrm{KMC}$ algorithm reproduces the decay in transition frequencies observed during QM/MD simulations.

Finally, we note that the current GRRM algorithm requires that IRC pathways be automatically calculated for all transition states $\mathrm{TS}_{i}^{m}$. In more complicated systems than those considered here, this would present a substantial inefficiency in the GRRM-KMC algorithm. Current research is therefore aimed at restricting the one-step GRRM search (step 1, Section II A) with a one-step ADDF search. Only a single IRC pathway calculation would then be necessary, once the pathway has been chosen (step 5, Section II A). Further research will also demonstrate the performance of the GRRM-KMC algorithm on more complicated (multistep, multipath) reactions. On the basis of the results presented here, we propose that the GRRM-KMC method is an attractive alternative for simulating complex nanoscale emergent phenomena, such as catalysis and self-assembly, which circumvents the infamous "time scale" problem encountered with traditional MD methods.

\section{ACKNOWLEDGMENTS}

A.J.P. and S.I. acknowledge support from the Australian Research Council (ARC No. DP140102894) and the Japan Society for the Promotion of Science (Open Partnership No. 13039901-000174). This work was supported in part by two CREST (Core Research for Evolutional Science and Technology) grants to S.I. from JST. S.I. and A.J.P. acknowledge support by the JSPS Sakura program for bilateral researcher exchange. I.M. acknowledges an Australian Postgraduate Award. The authors are grateful for generous supercomputing grants from The National Computational Infrastructure (NCI) National Facility and INTERSECT, Australia.

${ }^{1}$ B. J. Alder and T. E. Wainwright, J. Chem. Phys. 31, 459-466 (1959).

${ }^{2}$ A. Rahman, Phys. Rev. 136, A405-A411 (1964).

${ }^{3}$ Monte Carlo Methods in Statistical Physics, edited by K. Binder (Springer, Berlin, Heidelberg, 1986), Vol. 7, pp. 1-45.

${ }^{4}$ N. Metropolis, A. W. Rosenbluth, M. N. Rosenbluth, A. H. Teller, and E. Teller, J. Chem. Phys. 21, 1087-1092 (1953).

${ }^{5}$ E. Neyts and A. Bogaerts, Theor. Chem. Acc. 132, 1-12 (2012).

${ }^{6}$ E. C. Neyts, B. J. Thijsse, M. J. Mees, K. M. Bal, and G. Pourtois, J. Chem. Theory Comput. 8, 1865-1869 (2012). 
${ }^{7}$ K. M. Bal and E. C. Neyts, J. Chem. Phys. 141, 204104 (2014).

${ }^{8}$ A. F. Voter, Phys. Rev. B 57, R13985-R13988 (1998).

${ }^{9}$ R. W. W. Hooft, B. P. van Eijck, and J. Kroon, J. Chem. Phys. 97, 6690-6694 (1992).

${ }^{10}$ S. O. Samuelson and G. J. Martyna, J. Chem. Phys. 109, 11061-11073 (1998).

${ }^{11}$ A. Warmflash, P. Bhimalapuram, and A. R. Dinner, J. Chem. Phys. 127, 154112 (2007).

${ }^{12}$ G. M. Torrie and J. P. Valleau, J. Comput. Phys. 23, 187-199 (1977).

${ }^{13}$ A. F. Voter, Phys. Rev. Lett. 78, 3908-3911 (1997).

${ }^{14}$ R. A. Miron and K. A. Fichthorn, J. Chem. Phys. 119, 6210-6216 (2003).

${ }^{15}$ K. M. Bal and E. C. Neyts, J. Chem. Theory Comput. 11, 4545-4554 (2015).

${ }^{16}$ A. F. Voter, F. Montalenti, and T. C. Germann, Annu. Rev. Mater. Res. 32, 321-346 (2002).

${ }^{17}$ W. M. Young and E. W. Elcock, Proc. Phys. Soc. 89, 735 (1966).

${ }^{18} \mathrm{G}$. Henkelman and H. Jónsson, J. Chem. Phys. 115, 9657-9666 (2001).

${ }^{19}$ G. Henkelman and H. Jónsson, J. Chem. Phys. 113, 9978-9985 (2000).

${ }^{20}$ C. Peng and H. B. Schlegel, Isr. J. Chem. 33, 449-454 (1993).

${ }^{21}$ Y. Kumeda, D. J. Wales, and L. J. Munro, Chem. Phys. Lett. 341, 185-194 (2001).

${ }^{22}$ J. P. K. Doye and D. J. Wales, Z. Phys. D 40, 194-197 (1997).

${ }^{23}$ Y. Watanabe, S. Maeda, and K. Ohno, Chem. Phys. Lett. 447, 21-26 (2007).

${ }^{24}$ S. Maeda and K. Ohno, J. Phys. Chem. A 109, 5742-5753 (2005).

${ }^{25}$ S. Maeda and K. Ohno, Chem. Phys. Lett. 460, 55-58 (2008).

${ }^{26}$ K. Ohno and S. Maeda, J. Mol. Catal. A: Chem. 324, 133-140 (2010).

${ }^{27}$ S. Maeda and K. Ohno, Chem. Phys. Lett. 381, 177-186 (2003).

${ }^{28}$ K. Ohno and S. Maeda, Chem. Phys. Lett. 384, 277-282 (2004).

${ }^{29}$ G. H. Vineyard, Phys. Rev. 102, 981-992 (1956).

${ }^{30}$ G. Seifert and J.-O. Joswig, Comput. Mol. Sci. 2, 456-465 (2012).

${ }^{31}$ W. M. C. Foulkes and R. Haydock, Phys. Rev. B 39, 12520-12536 (1989).

${ }^{32}$ G. Zheng, S. Irle, and K. Morokuma, Chem. Phys. Lett. 412, 210-216 (2005).

${ }^{33}$ P. Koskinen and V. Mäkinen, Comput. Mater. Sci. 47, 237-253 (2009).

${ }^{34}$ Q. Cui, M. Elstner, E. Kaxiras, T. Frauenheim, and M. Karplus, J. Phys. Chem. B 105, 569-585 (2000).

${ }^{35}$ M. Gaus, Q. Cui, and M. Elstner, J. Chem. Theory Comput. 7, 931-948 (2011).

${ }^{36}$ M. Elstner, D. Porezag, G. Jungnickel, J. Elsner, M. Haugk, T. Frauenheim, S. Suhai, and G. Seifert, Phys. Rev. B 58, 7260-7268 (1998).

${ }^{37}$ G. Seifert, D. Porezag, and T. Frauenheim, Int. J. Quantum Chem. 58, 185-192 (1996).

${ }^{38}$ D. Porezag, T. Frauenheim, T. Köhler, G. Seifert, and R. Kaschner, Phys. Rev. B 51, 12947-12957 (1995).

${ }^{39}$ B. Aradi, B. Hourahine, and T. Frauenheim, J. Phys. Chem. A 111, 5678-5684 (2007).
${ }^{40}$ S. Nachimuthu, J. Gao, and D. G. Truhlar, Chem. Phys. 400, 8-12 (2012).

${ }^{41}$ A. Kumar and M. D. Sevilla, J. Phys. Chem. B 118, 5453-5458 (2014).

${ }^{42}$ B. V. Delchev, G. I. Shterev, and H. Mikosch, Chem. Mon. 139, 349-362 (2008).

${ }^{43}$ S. Sadhukhan, D. Muñoz, C. Adamo, and G. E. Scuseria, Chem. Phys. Lett. 306, 83-87 (1999).

${ }^{44}$ B. J. Smith and L. Radom, J. Phys. Chem. 99, 6468-6471 (1995).

${ }^{45}$ T. H. Dunning, J. Chem. Phys. 90, 1007-1023 (1989).

${ }^{46}$ E. Papajak, J. Zheng, X. Xu, H. R. Leverentz, and D. G. Truhlar, J. Chem. Theory Comput. 7, 3027-3034 (2011).

${ }^{47}$ M. J. Frisch, G. W. Trucks, H. B. Schlegel, G. E. Scuseria, M. A. Robb, J. R. Cheeseman, G. Scalmani, V. Barone, B. Mennucci, G. A. Petersson, H. Nakatsuji, M. Caricato, X. Li, H. P. Hratchian, A. F. Izmaylov, J. Bloino, G. Zheng, J. L. Sonnenberg, M. Hada, M. Ehara, K. Toyota, R. Fukuda, J. Hasegawa, M. Ishida, T. Nakajima, Y. Honda, O. Kitao, H. Nakai, T. Vreven, J. A. Montgomery, Jr., J. E. Peralta, F. Ogliaro, M. J. Bearpark, J. Heyd, E. N. Brothers, K. N. Kudin, V. N. Staroverov, R. Kobayashi, J. Normand, K. Raghavachari, A. P. Rendell, J. C. Burant, S. S. Iyengar, J. Tomasi, M. Cossi, N. Rega, N. J. Millam, M. Klene, J. E. Knox, J. B. Cross, V. Bakken, C. Adamo, J. Jaramillo, R. Gomperts, R. E. Stratmann, O. Yazyev, A. J. Austin, R. Cammi, C. Pomelli, J. W. Ochterski, R. L. Martin, K. Morokuma, V. G. Zakrzewski, G. A. Voth, P. Salvador, J. J. Dannenberg, S. Dapprich, A. D. Daniels, Ö. Farkas, J. B. Foresman, J. V. Ortiz, J. Cioslowski, and D. J. Fox, GaUssian 09, Gaussian, Inc., Wallingford, CT, USA, 2009.

${ }^{48}$ H. Kojima, A. Yamada, and S. Okazaki, J. Chem. Phys. 142, 174502 (2015).

${ }^{49}$ A. Yamada, H. Kojima, and S. Okazaki, J. Chem. Phys. 141, 084509 (2014).

${ }^{50}$ Y. Mori and Y. Okamoto, Phys. Rev. E 87, 023301 (2013).

${ }^{51}$ M. Kubillus, T. Kubař, M. Gaus, J. Řezáč, and M. Elstner, J. Chem. Theory Comput. 11, 332-342 (2015).

${ }^{52}$ M. Gaus, X. Lu, M. Elstner, and Q. Cui, J. Chem. Theory Comput. 10, 1518-1537 (2014)

${ }^{53}$ M. Gaus, A. Goez, and M. Elstner, J. Chem. Theory Comput. 9, 338-354 (2013).

${ }^{54}$ X. Lu, M. Gaus, M. Elstner, and Q. Cui, J. Phys. Chem. B 119, 1062-1082 (2015).

${ }^{55}$ L. Verlet, Phys. Rev. 159, 98-103 (1967).

${ }^{56}$ S. Nosé, J. Chem. Phys. 81, 511-519 (1984).

${ }^{57}$ W. G. Hoover, Phys. Rev. A 31, 1695-1697 (1985).

${ }^{58}$ G. Zheng, H. A. Witek, P. Bobadova-Parvanova, S. Irle, D. G. Musaev, R. Prabhakar, K. Morokuma, M. Lundberg, M. Elstner, C. Köhler, and T. Frauenheim, J. Chem. Theory Comput. 3, 1349-1367 (2007).

${ }^{59}$ Y. Ohta, Y. Okamoto, S. Irle, and K. Morokuma, Phys. Rev. B 79, 195415 (2009).

${ }^{60}$ Y. Ohta, Y. Okamoto, S. Irle, and K. Morokuma, ACS Nano 2, 1437-1444 (2008). 